Recepción: 07 / 10/ 2018

Aceptación: 20 / 11 / 2018

Publicación: 20 / 01 / 2019

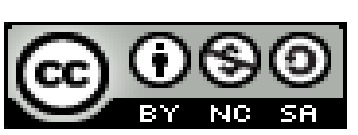

Ciencias de la salud

Artículo de Revisión

\title{
Recién nacidos prematuros de bajo peso: Seguimientos necesarios
}

\author{
Low birth premature newborns: Necessary follow-ups
}

\section{Recém-nascidos prematuros de baixo nascimento: Seguimentos necessários}

\author{
Angela R. Briones-Mera ${ }^{\mathrm{I}}$ \\ arbriones@utm.edu.ec \\ Krissia K. Saltarén-Pérez II \\ krissiasp@gmail.com \\ Leonardo J. Moreira-Rivas III \\ lexamori_91@hotmail.com \\ Jenniffer M. Chavez-Velasquez ${ }^{\text {IV }}$ \\ xtrememay@hotmail.com \\ Joffre P. Cevallos-Rosales V \\ paul_cevallos86@hotmail.com \\ Mario W. Pacheco-Moreira VI \\ mariowpacheco@hotmail.com
}

Correspondencia: arbriones@utm.edu.ec

I. Maestría en Investigación Clínica y Epidemiológica; Licenciada en Enfermería; Docente de la Universidad Técnica de Manabí; Facultad de Ciencias de la Salud; Escuela de Enfermería;, Portoviejo, Ecuador.

II. Médico en Funciones Hospitalarias; Hospital General Santo Domingo; Santo Domingo de Tsáchilas, Ecuador.

III. Médico; Hospital Dr. Gustavo Dominguez Zambrano - Área Ginecología - Medico General en Funciones Hospitalarias; Santo Domingo de Tsáchilas, Ecuador.

IV. Médico; Hospital General Santo Domingo - Medico General en Funciones Hospitalarias; Santo Domingo de Tsáchilas, Ecuador.

v. Médico; Hospital General Santo Domingo - Médico Residente; Santo Domingo de Tsáchilas, Ecuador.

vi. Médico; Hospital General Santo Domingo - Medico General en Funciones Hospitalarias; Santo Domingo de Tsáchilas, Ecuador. 


\title{
Resumen
}

En el año 2005 la OMS definió, después del Taller sobre “Optimización de la atención”, de denominar "Prematuro Tardío" a los nacidos entre las 34 semanas 0 días y 36 semanas 7 días después del inicio del último periodo menstrual. La inmadurez de las funciones orgánicas, las complicaciones y los trastornos específicos de la prematuridad determinan la susceptibilidad a una amplia variedad de enfermedades que difieren en comparación con los Recién Nacidos normales. Conociendo que la prematuridad y el bajo peso al nacer constituyen un problema para el mundo y que tienden a incrementarse a pesar de las acciones preventivas de salud y los avances en el cuidado intensivo perinatológico. En este caso nos proponemos no solo identificar las razones que aportan antes del nacimiento inherentes al periodo gestacional, sino esbozar cuales serían las consecuencias a enfrentar para y por el recién nacido para brindar la atención y cuidados necesarios para preservar la vida del nuevo ser. Se pretende hacer una revisión bibliográfica que sintetice la definición del recién nacido, luego evaluar la influencia del bajo peso en la vida del neonato. Lo primero que la metodología cualitativa nos muestra es que el criterio del investigador aporta en el destino del resultado final. Aunque el pronóstico y la supervivencia de los niños prematuros está relacionado directamente con la edad gestacional y peso al nacer, es importante la participación de los profesionales de la salud considerar la implementación de programas de seguimiento como objeto de investigación del recién nacido prematuro dado de alta, con el fin de verificar los efectos benéficos de las intervenciones tempranas en neonatos, los resultados pueden contribuir a evaluar las intervenciones y mejorar el cuidado.

Palabras claves: Recién Nacidos; Prematuro; Peso; Neonato.

\begin{abstract}
In 2005 the WHO defined, after the Workshop on "Optimization of care", to call "premature premature" those born between 34 weeks 0 days and 36 weeks 7 days after the start of the last menstrual period. The immaturity of the organ functions, complications and specific disorders of prematurity determine the susceptibility to a wide variety of diseases that differ compared to normal Newborns. Knowing that prematurity and low birth weight are a problem for the world and that tend to increase despite preventive health actions and advances in intensive perinatological care. In this case, we intend not only to identify the reasons that contribute before the birth inherent in the gestational period, but also to outline what the consequences would be for and for the newborn to
\end{abstract}


provide the necessary care and attention to preserve the life of the new being. It is intended to make a bibliographic review that synthesizes the definition of the newborn, then evaluate the influence of low weight in the life of the newborn. The first thing that the qualitative methodology shows us is that the criterion of the researcher contributes in the destiny of the final result. Although the prognosis and survival of premature infants is directly related to gestational age and birth weight, it is important for health professionals to consider the implementation of follow-up programs as an object of research for the premature newborn discharged., in order to verify the beneficial effects of early interventions in neonates, the results can help to evaluate interventions and improve care.

Keys words: Newly born; Premature; Weight; Neonate.

\section{Resumo.}

Em 2005, a OMS definiu, após o Workshop sobre "Otimização de cuidados", a designação de "prematuros prematuros" aqueles nascidos entre 34 semanas 0 dias e 36 semanas 7 dias após o início do último período menstrual. A imaturidade das funções do órgão, complicações e distúrbios específicos da prematuridade determinam a suscetibilidade a uma ampla variedade de doenças que diferem em comparação com os recém-nascidos normais. Sabendo que a prematuridade e o baixo peso ao nascer são um problema para o mundo e que tendem a aumentar, apesar das ações preventivas de saúde e dos avanços no cuidado perinatológico intensivo. Neste caso, pretendemos não apenas identificar os motivos que contribuem para o nascimento inerente ao período gestacional, mas também delinear quais seriam as conseqüências para e para o recém-nascido prestar o cuidado e a atenção necessários para preservar a vida da criança. novo ser. Pretende-se fazer uma revisão bibliográfica que sintetize a definição do recém-nascido, então avalie a influência do baixo peso na vida do recém-nascido. A primeira coisa que a metodologia qualitativa nos mostra é que o critério do pesquisador contribui no destino do resultado final. Embora o prognóstico e a sobrevida dos prematuros estejam diretamente relacionados à idade gestacional e ao peso ao nascer, é importante que os profissionais de saúde considerem a implementação de programas de acompanhamento como objeto de pesquisa para o recém-nascido prematuro e liberado., a fim de verificar os efeitos benéficos de intervenções precoces em neonatos, os resultados podem ajudar a avaliar intervenções e melhorar o atendimento.

Palavras chaves: Recém nascidos; Prematuro; Peso Neonato. 


\section{Introducción.}

En el año 2005 la OMS definió, después del Taller sobre "Optimización de la atención”, de denominar "Prematuro Tardío" a los nacidos entre las 34 semanas 0 días ( 239días ) y 36 semanas 7 días ( 259 días ) después del inicio del último periodo menstrual. Antiguamente denominados neonatos cerca del Término. Dado que estos pacientes representan alrededor del 70 al $75 \%$ de todos los prematuros, contribuyen sustancialmente a la mortalidad neonatal e infantil. La incidencia aumentó en los últimos tres años: 2006 15,7\%, 2007 16,6\% y 2008 17,1\% que corresponde a 1.341 niños prematuros tardíos. El 2004 en EEUU aumentó a 12,5 \% de nacidos prematuros menores de 37 semanas de edad gestacional. (Arandia \& Ayala, 2010)

Se consideran neonatos de alto riesgo los recién nacidos antes de término, con edad gestacional igual o menor a 36 semanas y con bajo peso al nacer si éste es inferior al percentil 10. El alto riesgo de morbilidad y mortalidad neonatal que se incrementa cuando el recién nacido tiene menor peso y menor edad gestacional implica proporcionarle atención especial. Los neonatos pretérmino, con bajo peso, cursan con múltiples problemas que se inician desde la etapa perinatal, luego durante la infancia e incluso hasta la edad adulta. Padecen más infecciones respiratorias y diarreicas que los prematuros de peso normal y discapacidades que van desde leves a graves, como: impedimentos físicos y mentales, limitaciones para la adaptación social debidas a alteraciones como la agresividad desafiante o de conducta. En Estados Unidos, 7.1\% de los recién nacidos vivos son pretérmino. (Martínez Contreras, Soria Rodríguez, Prince Vélez, Clark Ordoñez, \& Medina Ramírez, 2008)

Los prematuros tardíos son fisiológica y metabólicamente inmaduros. Una comprensión completa de la medida de inmadurez en estos niños es una patología poco estudiada. La probabilidad de que un 
prematuro tardío desarrolle al menos una complicación médica en el periodo neonatal 4 veces mayor que la de un niño a término. Tales problemas durante los primeros días incluyen:

1. Taquipnea transitoria

2. Déficit secundario de surfactante

3. Hipertensión pulmonar

4. Mayor requerimiento de asistencia respiratoria

5. Inestabilidad térmica

6. Hipoglucemia

7. Apnea8. Ictericia

8. Dificultades de alimentación

9. Signos clínicos transitorios difíciles de distinguir de unasepsis.

Un mayor riesgo de complicaciones a largo plazo, incluyen:

- Retraso en el desarrollo.

- Fracaso escolar.

- Trastornos del comportamiento

- Discapacidades sociales, identificados en los niños y adultos jóvenes (Arandia \& Ayala, 2010)

Entre los múltiples factores asociados al bajo peso al nacimiento (BPN) se han señalado las características antropométricas, nutricionales, socioculturales y demográficas de la madre; los antecedentes obstétricos y condiciones patológicas que afectan la funcionalidad y suficiencia placentaria, así como las alteraciones propiamente fetales. Debido a esta etiología multifactorial, las diversas investigaciones no han permitido dar un peso específico, ya sea asociativo o predictivo, 
para una u otra característica estudiada. Las repercusiones del BPN no se confinan sólo al período neonatal inmediato o al mediano plazo. El retardo en el crecimiento y desarrollo puede continuar hasta la edad adulta, e incluso manifestarse sobre la descendencia del afectado, de modo que la mortalidad por infecciones de adultos jóvenes que tuvieron BPN, puede llegar a ser hasta diez veces mayor, comparada con la de quienes tuvieron peso adecuado al nacimiento. Existe desde luego mayor riesgo de padecer episodios de enfermedad infecciosa aguda durante la infancia, lo que a su vez conlleva a desnutrición y consecuentemente al círculo vicioso infección/desnutrición/infección y a un incremento en la probabilidad de desarrollar enfermedades crónicas degenerativas durante la edad adulta. (Velázquez Quintana, Masud Yunes Zárraga, \& Ávila Reyes, 2004)

La inmadurez de las funciones orgánicas, las complicaciones y los trastornos específicos de la prematuridad determinan la susceptibilidad a una amplia variedad de enfermedades que difieren en comparación con los RN normales. Conociendo que la prematuridad y el bajo peso al nacer constituyen un problema para el mundo y que tienden a incrementarse a pesar de las acciones preventivas de salud y los avances en el cuidado intensivo perinatológico, se hace necesario continuar avanzando en el conocimiento para el logro de óptimos resultados y optimizar la atención integral de estos problemas, con el fin de aumentar no solo la tasa de sobrevida, sino la calidad de vida, y evitar las complicaciones y secuelas que hoy están presentes en este tipo de niños, sobre todo en los menores de 1500 g (Sarmiento Portal, Crespo Campos, Portal Miranda, Morales Delgado, \& Piloña Ruíz, 2009)

Es importante conocer las razones por las cuales se ve afectada la vida del neonato prematuro, en este caso nos proponemos no solo identificar las razones que aportan antes del nacimiento inherentes al periodo gestacional, sino esbozar cuales serían las consecuencias a enfrentar para y por el recién nacido para brindar la atención y cuidados necesarios para preservar la vida del nuevo ser, 
de manera tal que se minimice no solo los índices de mortalidad sino también los incides de morbilidad considerando los primeros años de vida hasta la adultez.

\section{Metodología.}

Se pretende hacer una revisión bibliográfica que sintetice la definición del recién nacido, luego evaluar la influencia del bajo peso en la vida del neonato. Se utilizaron frases y palabras clave para identificar los textos académicos y estudios publicados sobre los temas y enlazar finalmente en los resultados los cuidados y atenciones identificados a través de otras investigaciones que se hacen necesarias en los recién nacidos prematuros de bajo peso.

Lo primero que la metodología cualitativa nos muestra es que el criterio del investigador aporta en el destino del resultado final puesto que a medida que se utiliza en método deductivo se toma o rechaza la información que se busca.

Para el presente trabajo se ha enfocado en tres términos claves para las conclusiones que son, recién nacidos, prematuros y el bajo peso al nacer. De allí, los resultados expuestos.

\section{Resultados.}

Las causas del peso bajo son múltiples, entre ellas las enfermedades maternas, como: hipertensión arterial sistémica, diabetes mellitus, lupus eritematoso sistémico, alteraciones genéticas, malformaciones congénitas, infecciones perinatales, antecedentes de hijos con bajo peso al nacimiento, anormalidades placentarias y preeclampsia. (Velázquez Quintana, Masud Yunes Zárraga, \& Ávila Reyes, 2004) 
Angela R. Briones-Mera; Krissia K. Saltarén-Pérez; Leonardo J. Moreira-Rivas; Jenniffer M. Chavez-Velasquez; Joffre P. Cevallos-Rosales; Mario W. Pacheco-Moreira

La condición de los recién nacidos prematuros y/o con bajo peso al nacer constituye un enorme reto para los prestadores de servicios de salud, habida cuenta de la complejidad de procedimientos necesarios para lograr un aumento de peso armónico, regulación térmica y metabólica que garanticen un rápido progreso hacia la situación de normalidad fisiológica, propósitos de particular importancia en la atención de los recién nacidos con bajo peso, si se tienen en cuenta los riesgos que de este desequilibrio se desprenden hacia el desarrollo futuro de los niños. El Programa Madre Canguro, establecido en el Hospital San Rafael de Tunja desde el 1 de noviembre del 2007 propende por brindar apoyo a las madres mediante una capacitación que busca proporcionar bases conceptuales y prácticas que lleven a un correcto cuidado de sus hijos en el hogar; de este modo se busca mejorar las condiciones ambientales de desarrollo y maduración para el recién nacido (Lizarazo-Medina, Díaz, M., \& Ariza Riaño, 2012). Dicho programa se ha desarrollado en Colombia utilizando varias etapas educativas entre las madres y familias que decidieron participar, a través de talleres y actividades grupales se desarrolla una actividad principalmente educativa, informativa y concientizadora con repercusiones positivas y potenciadoras de la salud de los recién nacidos con bajo peso.

Para este proyecto es importante señalar algunos de sus resultados para conocer en cuales ámbitos se han centrado para mostrar un aporte.

\section{Ambito Socioeconómico y cultural:}

La edad promedio del grupo de madres fue 25,9 años. El 17,5 \%, eran menores de 18 años. El 24,7 $\%$, no contaban con pareja estable y el 13,7 \%, contaba con algún tipo de apoyo familiar.

264 madres (71\%), tienen por ocupación las actividades de ama de casa. La calidad de la nutrición en la familia, el 47,3\%, es insuficiente. 
En ingreso familiar promedio mensual ascendió a \$ 501 984, es importante anotar que apenas $25 \%$, de las familias tiene ingresos mensuales superiores a 500000 pesos mensuales 42,6\%, de las familias no cuenta con agua potable en la casa $61,1 \%$, de las madres debía desplazarse durante 1 hora o más tiempo para llegar al Hospital.

\section{Antecedentes maternos y obstétricos:}

$3,2 \%$, de las maternas reportaron ser fumadoras y $51(13,7 \%)$, consumían alcohol con frecuencia de ocasional a frecuente. El promedio de embarazos por materna fue 2,45, 30,5\%, de las mujeres tenían 3 o más embarazos incluyendo el actual. El promedio de controles prenatales fue 5,4, apenas 1,6 \%, no tuvieron controles. 40,6 \% de los embarazos fueron no deseados.

En cuanto a las patologías durante el embarazo, noventa y siete mujeres $(26,1 \%)$, presentaron cuadros de Toxemia Gravídica y presentaban anemia 37,5 \% de la muestra.

\section{Sufrimiento fetal:}

Se reportó frecuencia cardiaca fetal anormal en 52 casos (13,9\%) y Monitoreo anormal en 67 (18 \%). En 34 casos se encontró líquido amniótico meconiado $(9,1 \%)$ y Ruptura prematura de membranas en $106(28,4 \%)$.

\section{Parto y adaptación neonatal:}

El parto vía vaginal ocurrió en $51,7 \%$; por cesárea 48,0 \%, de las cuales solo $6,2 \%$, fueron electivas.

La escala de Apgar al minuto del nacimiento fue igual o inferior a tres en 4 casos $(1,1 \%)$, es importante anotar que en 16,1 \% de los nacimientos se omitió anotar pa prueba de Apgar. El Apgar 
promedio al minuto del nacimiento fue de $7,7(\mathrm{SD}=1,23)$. En 36 casos $(9,7 \%)$, hubo necesidad de maniobras de reanimación. Es importante anotar que solo un bebé registró a los 5 minutos Apgar igual o weight in United Arab Emirates. Journal of Biosocial Science1996; 28:339-346. inferior a 4, por cuanto este es un criterio estandarizado de asfixia, que define el pronóstico del niño.

A los cinco minutos del nacimiento el APGAR promedio fue 9,09 ( $\mathrm{SD}=1,07)$; igual o inferior a tres en un caso $(0,3 \%)$, entre cuatro y siete en 22 casos $(5,9 \%)$, e igual o superior a ocho en $292(48,4$ $\%)$, no aparece reporte en 58 casos (15,5\%).

El peso promedio al momento del nacimiento fue de 1969 gramos ( $\mathrm{SD}=401,25)$, no se observaron diferencias estadísticamente significativas por sexo. (Lizarazo-Medina, Díaz, M., \& Ariza Riaño, 2012)

Dentro de los alcances más relevantes obtenidos mediante la metodología Madre canguro se han destacado la ganancia de peso a un ritmo uniforme y sostenido, la mejor calidad de atención y cuidado proporcionada al recién nacido, el fortalecimiento de la relación madre-hijo y la reducción significativa en los costos de la atención demostrable en la reducción de la mortalidad, la incidencia de complicaciones severas, infecciones nosocomiales, adecuación de la lactancia materna exclusiva y reducción de la disconformidad materna. Un aspecto relevante de los beneficios del PMC tiene que ver con el fortalecimiento de la relación madre - hijo, en particular con el fortalecimiento de la seguridad y confianza de las mujeres madres de bebés prematuro o con bajo peso al nacer, ya que permite manifestar las preocupaciones referidas al estado de salud y adquirir respuestas concretas acerca de la manera de ayudar en el cuidado de su hijo, lo que influye favorablemente sobre el estado de ansiedad (Lizarazo-Medina, Díaz, M., \& Ariza Riaño, 2012) 
Estos niños son extremadamente vulnerables a las infecciones que, se plantea, son unas de las complicaciones principales. Siempre se debe tener en cuenta la necesidad actuación, una atención diferenciada con el personal de enfermería para la los cuidados utilizando poco la ventilación asistida y en la alimentación se usa exclusivamente leche materna. Algunos autores, como Mc Court(1998) y Fleisher (1996), justifican la elevada incidencia de sepsis en estos niños tan pequeños e inmaduros, condicionadas por la infección perinatal, la inmadurez del sistema inmunitario, la disminución de las defensas naturales, la prolongada hospitalización, las complicaciones iatrogénicas relacionadas con la terapia intensiva y los estados prolongados, dada la enfermedad de base. Si tenemos en cuenta las características especiales de estos pequeños seres, cuántos factores determinan que puedan sobrevivir o no, las condiciones del parto, el tratamiento inmediato, la profilaxis de la asfixia, del enfriamiento, los trastornos metabólicos, la respiración adecuada, la profilaxis de las infecciones y, si somos capaces de brindarles al conocer de la eminencia de un parto en un niño extremadamente inmaduro los cuidados dirigidos a evitar estos trastornos, entonces podemos decir que estamos actuando bien. Y si aun somos capaces de brindarles en el servicio de neonatología los cuidados gentiles que este niño demanda, entonces podemos decir que seguimos actuando bien. Y si además logramos mejorar su calidad de vida, pues entonces podemos decir que aun seguimos actuando bien. Tudehope, Burns, Gray, Mohay, y Roger (1995) reportan la importancia de estos cuidados y plantean que la principal causa de muerte de estos niños es la sepsis, cuyos mecanismos etiológicos están detallados anteriormente, la enfermedad de membrana hialina tuvo una alta letalidad también. Esta y la hemorragia intracraneal fueron las principales causas de muerte. En todos los casos la edad de fallecimiento fue menor de los 7 días. (Sarmiento Portal, Crespo Campos, Portal Miranda, Morales Delgado, \& Piloña Ruíz, 2009) 
Angela R. Briones-Mera; Krissia K. Saltarén-Pérez; Leonardo J. Moreira-Rivas; Jenniffer M. Chavez-Velasquez; Joffre P. Cevallos-Rosales; Mario W. Pacheco-Moreira

Un niño preternimo puede presentar problemas de desarrollo por tanto se señala lsa distintos problemas a los que se pueden enfrentar aparte del bajo peso sea este consecuencia o causa de las demás complicaciones.

\section{Dificultad Respiratoria}

Los recién nacidos prematuros nacen durante el desarrollo pulmonar en la etapa de transición del período de saco Terminal (período alveolar). Durante la etapa de saco ter-minal, la unidades respiratorias se componen de sáculos alveolares alineados con el tipo II cúbicas (fuente de surfactante) y tipo plano I las células epiteliales. Deficiencias funcionales en surfactante y gestión del agua de pulmón.

\section{Apnea}

La incidencia de la apnea en los prematuros tardíos es de 4\% a 7\%, significativamente menor que en los recién nacidos extremadamente prematuros, pero significativa-mente mayor que en recién nacidos a término ( $1 \%$ a $2 \%)$. La predisposición a la apnea se produce debido a una mayor susceptibilidad a la depresión respiratoria hipóxica, la disminución de quimio sensibilidad central al dióxido de carbono, inmadurez del sistema nervioso central, los cerebros de estos niños son significativamente más pequeños, menos mielinizados, y contienen menos circunvoluciones que los surcos de recién nacidos a término.

\section{Regulación de la Temperatura}

La termorregulación de los prematuros tardíos se ve comprometida por los bajas cantidades de grasa marrón y blanco, la función hipotalámica inmadura y bajas concentraciones de hormonas responsables del metabolismo de la grasa parda (como prolactina, leptina, noradrenalina, 
triyodotironina, y cortisol). Durante el estrés por frío que sigue al nacimiento, la hipotermia se experimenta con mayor frecuencia a finales de los recién nacidos prematuros de recién nacidos a término debido a una menor capacidad para generar calor adiposo marrón y menor depósitos de grasa blanca para el aislamiento de la frío. Metabolismo de la Glucosa. La incidencia de la hipoglucemia se correlaciona inversamente con la edad gestacional. Los neonatos prematuros tardíos, están predispuestos a la hipoglucemia debido a la inmadurez de la glucogenólisis hepática y la lipólisis del tejido adiposo, alteración de la regulación hormonal, la gluconeogénesis hepática deficiente y la cetogénesis, y las reservas de glucosa baja. Metabolismo de la bilirrubina La ictericia es más frecuente en los prematuros tardíos que los recién nacidos a término. La duración de la ictericia esa menudo más prolongado, y las concentraciones máximas de bilirrubina indirecta con frecuencia son más altos que los encontrados en el a término. Debido al retraso de la maduración y la menor concentraciones de glucuronil-transferasa diphosphoglucuronate uridina, La circulación entero hepática de la bilirrubina también contribuye a la elevación de la bilirrubina.

\section{Alimentación y función gastrointestinal}

El comportamiento alimenticio y la función gastrointestinal son inmaduros en los recién nacidos prematuros. Pue-den tener dificultades en la alimentación por el tono motor bajo, la falta de coordinación en la secuencia de la succión, la deglución y la dismotilidad del aparato digestivo. Desarrollo del cerebro y desarrollo a largo Plazo, Educación, comportamiento, y la función social. El desarrollo del cerebro avanza en el feto y hasta la in-fancia. El cerebro pesa en prematuros tardíos alrededor de dos tercios de un recién nacido a término, tiene un número significativamente menor circunvoluciones y surcos, y es menos mielinizado, lo que significa que tiene un efecto negativo en el desarrollo del cerebro y las complicaciones de parto prematuro puede contribuir al desarrollo, conductuales, educativas, y las discapacidades sociales descritos en prematuros casi a término. El 
trastorno de déficit de atención es de 1,7 veces más frecuente en los prematuros tardíos que en los recién nacidos a término. Por otra parte, en una cohorte grande de Suecia adultos jóvenes, el nacimiento prematuro tardío se asoció con mayores tasas de discapacidad y la necesidad de apoyo social y menores tasas de educación post-se-cundaria y empleo. La cohorte sueca de prematuros tardíos y principios de los bebés plazo (definida en 33 a 36semanas de gestación y gestación 37 a 38 semanas, respectivamente) significativamente contribuye a las tasasgeneral de discapacidad en la Población de Suecia; más del74\% de las discapacidades se producen en la cohorte de gestación de 33 a 38 semanas. En Noruega los adultos entre 20 y 36 años de edad que nacieron como prematuro tardío también tenían tasa más alta de morbilidad: parálisis cerebral (2,7 veces), discapacidad intelectual (1,6veces), trastornos del desarrollo psicológico, comporta-miento, y la emoción (1,5 veces), esquizofrenia (1,3veces), otras discapacidades graves (1,5 veces) y cualquier incapacidad médica que afecta gravemente la capacidad de trabajo (1,4 veces) que recién nacidos a término (Arandia \& Ayala, 2010)

\section{Problemas nutricionales de los recién nacidos con BPN}

Muchos de los factores etiológicos y de las alteraciones asociadas a BPN tienen un efecto importante sobre la nutrición, lo que impide una recuperación adecuada. Los prematuros son especialmente susceptibles para desarrollar desnutrición adquirida, sobre todo si se encuentran críticamente enfermos en el período postnatal. Los problemas asociados a hipoxia que pueden favorecer la presencia de enterocolitis necrosante son comunes en los prematuros y en los niños de término, exacerbados por el afán médico de administrar una alimentación hipercalórica temprana.

La meta de la alimentación neonatal es imitar el crecimiento y la composición corporal intrauterinos en el prematuro y recuperar e igualar la velocidad de crecimiento postnatal. El feto tiene como su 
principal aporte nutricio la glucosa que toma de la madre a través de la placenta, con un consumo mínimo de grasas. Otra posibilidad de nutrición de productos con BPN, sobre todo prematuros y con menos de $1500 \mathrm{~g}$ es la alimentación enteral mínima. El incremento debe ser paulatino y de preferencia con leche materna que tiene efecto protector contra enterocolitis necrosante.68 $\mathrm{Su}$ finalidad principal es "preparar" al intestino para favorecer la producción de hormonas y péptidos, favorecer la maduración de la peristalsis y de la mucosa intestinal. Recientes investigaciones en prematuros desde la Conferencia Congreso Internacional de Pediatría (2001) han evaluado la administración temprana de probióticos (especies bacterianas no patógenas), encontrando un efecto preventivo contra enterocolitis necrosante por una probable regulación de la microflora intestinal.

Las fórmulas para prematuros basadas en leche de vaca tienen inferioridad nutricional en comparación con la leche materna. La leche fresca materna de pretérmino contiene más proteínas, minerales y valor energético que la leche humana de término; sin embargo, esta diferencia persiste sólo unas dos semanas y a partir de este momento la leche pretérmino puede considerarse inadecuada, sobre todo para los prematuros de MBP, con una acelerada velocidad de crecimiento. Se ha intentado "fortificar" la leche materna con productos derivados de leche materna o bovina con diferentes composiciones, pero los resultados son poco concluyentes en términos de recuperación del crecimiento después del alta. Otros investigadores han promovido la adición de ácidos grasos poliinsaturados de cadena larga a las fórmulas lácteas para modificar la concentración de éstos a nivel cerebral, de la misma manera que sucede con la alimentación al seno materno, pero aún se desconocen las consecuencias de esta medida a largo plazo.

Independientemente de la edad gestacional y peso al nacimiento, a todo niño con BPN debe ofrecérsele aportes adecuados de hierro, cobre, zinc y vitaminas para recuperar los depósitos que no fueron acumulados in útero. La deficiencia de este mineral durante las primeras etapas del desarrollo 
cerebral se ha relacionado con alteraciones de la conducta que incluyen deficiencias del aprendizaje y de la memoria. Los cálculos para una alimentación adecuada en el período neonatal son un ejercicio de paciencia y de constante cambio que dependerán en gran medida de las situaciones individuales y la disposición de elementos a nuestro alcance. (Velázquez Quintana, Masud Yunes Zárraga, \& Ávila Reyes, 2004)

\section{Leche materna para el prematuro}

A pesar de la insuficiencia demostrada de algunos nutrientes como proteínas, calcio, fósforo, zinc, hierro, sodio y algunas vitaminas, la leche materna es el alimento que se prefiere para lactantes en extremo prematuros y para el prematuro en general, a causa de su composición, biodisponibilidad aumentada de nutrimentos, propiedades inmunitarias y la presencia de hormonas, enzimas y factores de crecimiento. La magia está en la forma de extraerla, conservarla, manipularla y administrarla al recién nacido, cómo complementarla con los nutrientes deficitarios y determinar hasta cuándo es necesaria esa supleción.

Lo ideal, es que la madre pueda entrar al departamento cada $3 \mathrm{~h}$ para extraer de forma directa la leche para su hijo. La leche materna directa tiene ventajas sobre la leche de banco, incluso cuando se logre tener un banco de leche materna de prematuro con similitud de edades gestacionales. La leche materna directa aporta todos los macronutrientes con las características propias de su edad gestacional. Se ha demostrado que la leche de pretérmino tiene un mayor tenor de proteínas que la de término, y aporta los aminoácidos esenciales y condicionalmente esenciales como la taurina, carnitina, cisteína y tirosina, con menor composición de aminoácidos aromáticos; además ofrece la ventaja de mantener intactos los factores inmunológicos celulares. 
La leche pasteurizada a $62,5^{\circ} \mathrm{C}$ durante 30 min presenta una reducción parcial del contenido de lactoferina, lisozima e IgA, con pérdida de algunas células de defensa y preservando los demás factores. A pesar de la reducción del $30 \%$ de la cantidad total de IgA, su valor biológico se mantiene inalterado según el estudio de Carbonare y Carneiro Sampaio.15

\section{Ventajas de la leche materna para el pretérmino:}

A) Valor nutricional

- Mayor contenido de proteínas y de sodio.

- Aporte de aminoácidos esenciales y condicionalmente esenciales (taurina, cisteína, carnitina y tirosina)

- Moderado aumento en el contenido en energía y grasa.

- Aporte de ácidos grasos esenciales elongados con una proporción adecuada, que mejora la composición de las membranas celulares del cerebro, retina y los eritrocitos.

- Concentraciones adecuadas de colesterol para la formación de membranas

- Alta biodisponibilidad de hierro (40-50\%)

- Mejor relación Ca:P (2:1) que favorece la absorción

B) Ventajas para el sistema gastrointestinal y renal

- Baja carga de solutos

- Presencia de lipasa estimulada por sales biliares que ayuda a la digestión intestinal de las grasas

- Mayor vaciamiento gástrico

- Presencia de factores tróficos y de maduración sobre el sistema gastrointestinal. 
Angela R. Briones-Mera; Krissia K. Saltarén-Pérez; Leonardo J. Moreira-Rivas; Jenniffer M. Chavez-Velasquez; Joffre P. Cevallos-Rosales; Mario W. Pacheco-Moreira

- Mejor absorción de las grasas por su actividad lipolítica

C) Factores inmunológicos

- Presencia de oligosacáridos que participan en el sistema defensivo

- Factores inmunológicos que previenen enterocolitis necrotizante.

D) Mejor relación madre-infante temprana a través del "Plan Canguro", con mejor estimulación del desarrollo psicomotor.

\section{Componentes de la leche humana que pueden ejercer funciones beneficiosas de carácter no nutritivo:}

- Bifidobacterias: inhiben el crecimiento de gérmenes patógenos entéricos.

- Inhibidores del metabolismo patógeno de los microbios: la lactoferrina y las proteínas que enlazan los folatos y la vitamina B 12 impiden el crecimiento de gérmenes in vitro .

- Enzimas: lisozimas, peroxidasas con actividad bacteriostática y otras enzimas que actúan en el transporte y síntesis mamarios de los componentes de la leche materna o en los procesos de digestión y metabolismo de recién nacidos.

- Otros agentes antiinfecciosos: factores estimuladores de la proliferación de colonias de leucocitos como, granulocitos y macrófagos, o ambos, y la fibronectina que facilita la función de los fagocitos.

- Inmunoglobulinas: fundamentalmente la Ig A secretora con mayor concentración en el calostro que en la leche definitiva, la cual protege frente a gérmenes del tracto gastrointestinal de la madre. IgM, IgG, IgE, IgD, complemento (C3 y C4). 
- Agentes inmunomoduladores: prolactina, IgA secretora, prostaglandina E 2 y algunas citoquinas

- Péptidos: derivados de la caseína que regulan la motilidad gastrointestinal y otros como el péptido inhibidor de la gastrina y la bombesina, que actúan sobre el crecimiento, maduración y regulación gastrointestinal.

- Lípidos: ciertos ácidos grasos de cadena media y los mono y poliinsaturados ejercen una actividad antivírica, antibacteriana y antifúngica.

- Factores de crecimiento: factor de crecimiento epidérmico y factores de crecimiento semejantes a la insulina. Ambos favorecen la proliferación celular del epitelio intestinal y tienen actividad antiinflamatoria.

- Hormonas tiroideas.

- Somatostatina: tiene propiedades inmunosupresoras y antiinflamatorias en el tracto gastrointestinal del lactante. (Díaz, 2005)

\section{Leche materna en el crecimiento intrauterino retardado}

Los recién nacidos con crecimiento intrauterino retardado,.Existe disminución del número de células de intestino y páncreas con disminución de su peso, reducción del contenido enzimático del páncreas, disminución de las disacaridasas totales, enteroquinasa y fosfatasa alcalina, y de la absorción de grasas y proteínas, calcio, fosfato y vitaminas liposolubles.

El riesgo elevado de enterocolitis necrotizante no permite la alimentación con altos volúmenes de leche y por tanto en este grupo de pacientes la alimentación enteral mínima con leche materna puede considerarse una terapéutica. 
Angela R. Briones-Mera; Krissia K. Saltarén-Pérez; Leonardo J. Moreira-Rivas; Jenniffer M. Chavez-Velasquez; Joffre P. Cevallos-Rosales; Mario W. Pacheco-Moreira

Los beneficios de una lactancia exitosa han sido bien documentados por los estudios a largo plazo sobre el desarrollo visual de Carlson (1996), O`Connor (2001) y Uauy (1996) y los estudios del desarrollo cognitivo de Dewey (2001) En todos ellos los resultados de los mejores índices se relacionaron con los grupos que mantuvieron lactancia materna por más de 4 meses de edad.

La lactancia materna es sin dudas la mejor alimentación para el niño menor de 6 meses de edad. Hasta el momento, y a pesar de los esfuerzos de las diferentes industrias de alimentos, no se ha logrado ningún producto que pueda sustituir, ni sus bondades ni el calor materno al ofrecerla. (Díaz, 2005)

\section{Aspectos a considerar en el manejo del recién nacido prematuro}

\section{Valoración clínica de enfermería por patrones funcionales de salud}

1. Percepción y control de la salud. En esta población se ha observado que la mayoría de padres de prematuros provienen de nivel socioeconómico bajo, por ende las madres carecen de asistencia médica prenatal, nutrición deficiente, y enfermedades infecciosas no tratadas. En neonatos prematuros, la sepsis y la meningitis son cuatro veces más frecuentes debido a la necesidad de aplicar medios invasivos (catéteres, tubos, venoclisis, etcétera) para conservar y recuperar la salud del paciente, asimismo sus mecanismos de defensa son inferiores.

2. Nutricional y metabólico. El recién nacido prematuro generalmente tiene un peso inferior a $2500 \mathrm{~g}$; tiene piel fina, brillante rosa o rojiza, escasa cantidad de grasa subcutánea y pelo, cartílago auditivo externo no desarrollado, escasa coordinación de los reflejos de succión y deglución, principalmente en pequeños menores de 34 semanas de gestación, la superficie corporal es grande en relación a su masa corporal, la grasa parda es escasa para mantener su temperatura corporal, poca implantación de cabello en el cuero cabelludo, etcétera. Estos pequeños en la unidad de estudio son 
nutridos principalmente a través de alimentación forzada, midiendo residuo gástrico, ya que esto científicamente da mejores resultados y menos accidentes.

3. Eliminación. La función renal y pulmonar son inmaduras, lo que disminuye la capacidad para concentrar la orina, aumentando la probabilidad de retención de líquidos y trastornos electrolíticos, así como trastornos respiratorios, hipomotilidad intestinal, etcétera.

4. Actividad y ejercicio. Actividad espontánea, tono muscular disminuido, las extremidades no mantienen su posición flexionada. A nivel respiratorio colapso alveolar y atelectasia debido a la baja producción de surfactante, datos característicos de inmadurez del centro respiratorio, responsables de crisis de apnea, dando lugar a requerir apoyo ventilatorio.

1. Desarrollan con mayor frecuencia hiperbilirrubinemia y kernicterus, frecuencia cardiaca baja, anemia, llanto débil y puede presentar taquipnea transitoria. El sistema inmunitario es inmaduro, de 30 a $90 \%$ de recién nacidos antes del término presentan hipocalcemia e hipoglicemia.

5. Reposo y sueño. Letargo, sueño interrumpido por maniobras frecuentes, sobresaltos.

6. Cognoscitivo y perceptual. Puede haber presencia de crisis convulsivas como consecuencia de hemorragia cerebral, presentan fontanelas amplias, existe desconocimiento por parte de los padres de las necesidades del recién nacido y cómo atenderlas.

7. Autopercepción y autoconcepto. Existe preocupación de los padres y temor para el cuidado del recién nacido prematuro, autoestima baja.

8. Función y relación. Es frecuente que el estado civil de los padres de neonatos prematuros es la soltería.

9. Sexualidad y reproducción. En los varones, genitales pequeños, subdesarrollados y los testículos sin descenso. En las niñas los labios mayores no cubren aún los menores. 
Angela R. Briones-Mera; Krissia K. Saltarén-Pérez; Leonardo J. Moreira-Rivas; Jenniffer M. Chavez-Velasquez; Joffre P. Cevallos-Rosales; Mario W. Pacheco-Moreira

10. Afrontamiento y tolerancia al estrés. El estrés por frío del recién nacido prematuro agota con rapidez las reservas de grasa originando incremento en las necesidades metabólicas, consumo de oxígeno, acidosis metabólica, hipoxemia e hipoglicemia. Por lo regular los padres expresan la incapacidad para cuidar al recién nacido, lo que puede originar crisis, que se manifiesta por conducta inadaptada, ansiedad, depresión y síntomas somáticos.

11. Valores y creencias. Mitos y creencias de los padres relacionados con el cuidado del recién nacido.

Diagnósticos de enfermería de acuerdo a la NANDA.

1. Deterioro del intercambio gaseoso

2. Patrón respiratorio ineficaz

3. Termorregulación ineficaz

4. Riesgo de deterioro de la integridad cutánea

5. Riesgo alto de alteración de los procesos de eliminación

6. Patrón de alimentación ineficaz del lactante

7. Riesgo de infección

8. Temor

9. Déficit de conocimientos

Aquí se muestran los diagnósticos, las intervenciones de enfermería, la fundamentación científica y los criterios de resultados que son considerados para los siguientes eventos patológicos del prematuro. Con este conjunto de acciones y cuidados sistemáticos, se pretende dar respuesta oportuna que asegure la calidad de la atención a estos pacientes. 


\section{Problemas interdependientes}

Se describen las intervenciones y criterios de manejo de los siguientes eventos secundarios del recién nacido prematuro.

1. Hiperbilirrubinemia

2. Riesgo de hipoxia

3. Riesgo de acidosis metabólica

4. Anemia

5. Riesgo de septicemia

\section{Plan de alta del recién nacido prematuro}

Las características socioeconómicas y culturales de los pacientes prematuros pueden incidir en la sobrevida de los pacientes. Es por ello que el plan de alta será determinante en el progreso y estabilización a la vida del prematuro. Este es el gran reto de los profesionales de enfermería, en donde la educación y entrenamiento que se dé a los padres en el manejo de su hijo, traerá consigo buenos o malos resultados en la salud. Estas acciones se evitarán los reingresos a los hospitales por otras afecciones por el mal manejo del paciente. El desafío del cuidado es coadyuvar a mejorar la calidad de vida minimizando la patología agregada como las infecciones y lograr un desarrollo neurológico que le permita integrarse a la vida familiar y a la sociedad a través de la termorregulación, programa de resucitación neonatal, terapia con surfactante, oxigenación, terapia con esteroides y cuidados especiales del neonato prematuro esenciales para el crecimiento, desarrollo y sobrevivencia. (Mata, Salazar, \& Herrera, 2009) 


\section{Conclusiones.}

El bajo peso al nacimiento (BPN) es un problema de salud pública mundial, que impacta en gran medida sobre la mortalidad neonatal e infantil en menores de un año, particularmente en el período neonatal. Cerca de $90 \%$ de los nacimientos de productos con bajo peso ocurren en países en vías de desarrollo para una prevalencia global de 19\%. En los países del sur asiático, el BPN alcanza hasta $50 \%$, en comparación con América Latina donde ocurre en aproximadamente 11\%, mientras que para los países desarrollados se ha considerado por debajo de 6\%. (Velázquez Quintana, Masud Yunes Zárraga, \& Ávila Reyes, 2004)

El bajo peso al nacer es una condición clínica que significa un riesgo sustancialmente elevado de morir, hasta 14 veces mayor para los bebés. Los factores generales que se encuentran asociados a él, además de la pobreza, son el bajo nivel de escolaridad, embarazo adolescente, malnutrición de la madre, tabaquismo, presencia de hipertensión arterial durante el embarazo, embarazo múltiple, anemia de la madre, antecedente de aborto inducido, infecciones cérvico-vaginales, incompetencia ístmico-cervical. También se ha reportado que a medida que aumenta la edad materna el peso de los recién nacidos tiende a disminuir. A este respecto es importante anotar que la identificación temprana y tratamiento de complicaciones durante el embarazo como las vaginosis y también la administración de calcio suplementario, hierro y folato se identifican como intervenciones oportunas y capaces de disminuir sustancialmente el riesgo de partos pre término o recién nacidos con bajo peso al nacer. Se ha demostrado la existencia de una fuerte asociación de la condición de bajo peso al nacer con la ocurrencia más frecuente durante los primeros 12 meses de vida, complicaciones severas, entre los que destacan retraso del desarrollo neurológico, hemorragia cerebral, alteraciones respiratorias y otras que imponen la necesidad de atención prolongada y hospitalización. También existe sólida evidencia que prueba la asociación entre el bajo peso al nacer y mayor mortalidad 
infantil. Estudios epidemiológicos más recientes muestran una posible asociación entre el BPN y enfermedades del adulto tales como la hipertensión arterial y la diabetes mellitus no insulinodependiente (Lizarazo-Medina, Díaz, M., \& Ariza Riaño, 2012)

En México, en el Instituto Nacional de Perinatología, 8 a 9\% de los nacimientos son de prematuros. La información de 1999 a 2005 del Banco de Datos Delegacional, subsistema 10 del Hospital de Gineco-Pediatría con Medicina Familiar núm. 31 del IMSS, señala que en 1999 hubo 6,500 nacimientos, de los que $6.5 \%$ correspondió a neonatos pretérmino, este último porcentaje se incrementó a $7.5 \%$ por año hasta el 2005. La proporción de recién nacidos pretérmino con bajo peso al nacer, en 1999, en nuestro hospital, fue de 5.3\%, cifra que se incrementó para el 2002 a $7 \%$ y para el 2005 a 10.5\%. En Estados Unidos se reporta la ocurrencia de enfermedad hipertensiva en 12 a $22 \%$ de las mujeres embarazadas, que ocasiona $17.6 \%$ de las muertes maternas y $15 \%$ de la mortalidad perinatal. En América Latina la morbilidad perinatal es de 8 a $45 \%$ y la mortalidad perinatal de 1 a $33.3 \%$. La preeclampsia se reporta en 3 a $8 \%$ de los embarazos y se considera una enfermedad irreversible que afecta múltiples órganos y lesiona al feto y a la madre. Es un problema de salud pública que aumenta: las enfermedades maternas durante el embarazo, la proporción de neonatos prematuros y de peso bajo al nacer, la morbilidad y mortalidad, y el gasto generado por la atención médica de la madre y su hijo. (Martínez Contreras, Soria Rodríguez, Prince Vélez, Clark Ordoñez, \& Medina Ramírez, 2008)

El predominio del sexo masculino encontrado coincide con los reportes de otros autores, pues la literatura médica señala que muchas de las afecciones frecuentes en este tipo de niños tienen lugar en este sexo. Sin embargo, algunos autores reportan un predominio de ingresos de niños del sexo femenino. González Cabello y cols. (2007) encontraron en su estudio una edad gestacional promedio de 30 semanas y un peso promedio de $1100 \mathrm{~g}$; similares resultados son los reportados por 
Santamaría en México13 y Acevedo en Santiago de Cuba. Por su parte, estudios en Costa Rica encontraron también predominio de edad gestacional alrededor de 30 semanas y coincide con esta investigación en cuanto al grupo de peso. La sobrevida de los prematuros de muy bajo peso ha experimentado una mejoría significativa en las últimas décadas. En Sudamérica los centros que integran el grupo Neocosur (Neonatología Cono Sur) realizaron un estudio prospectivo que incluyó 385 recién nacidos menores de 1500 g provenientes de 11 centros de 4 países (Chile, Argentina, Perú y Uruguay) y encontraron una sobrevida global del grupo del $73 \%{ }^{7}$ En Chile, la sobrevida para los niños entre 1000 y $1499 \mathrm{~g}$ es del 77,1\%, mientras que para recién nacidos entre 500 y $900 \mathrm{~g}$ es sólo del 34,4 \%. (Sarmiento Portal, Crespo Campos, Portal Miranda, Morales Delgado, \& Piloña Ruíz, 2009)

El BPN es un problema presente, con importantes repercusiones para el futuro de la sociedad, es indispensable identificar los factores de riesgo involucrados para cada región y estrato ponderal para definir correctamente las políticas de salud que permitan incidir positivamente sobre los riesgos de enfermedad y muerte para nuestra población. Debe existir un apoyo sostenido para los programas que ya se realizan para mejorar el estado nutricional de la población en general y en particular de las mujeres en edad de procreación, así como mejorar la atención de las mujeres gestantes y en período de lactancia. Es importante reconocer que ahora el bajo peso debe ser analizado en forma estratificada, ya que la etiología y condiciones al nacimiento y por lo tanto la morbimortalidad son diferentes para cada grupo particular. Asimismo, como punto clave, aunque fuera del contexto médico, habrá que invertir para mejorar el nivel socioeconómico de la población. El BPN es una enfermedad para toda la vida y debe ser planteado de esa manera para establecer correctamente su tratamiento integral. (Velázquez Quintana, Masud Yunes Zárraga, \& Ávila Reyes, 2004) 
Aplicar la teoría de Marjory Gordon para valoración del neonato prematuro implica considerar el método de patrones funcionales, que contempla al paciente, la familia y su entorno de manera global y holística, permite reunir todos los datos necesarios para abordar los problemas de salud reales y potenciales a través de actividades independientes e interdependientes a realizar por los profesionales de enfermería. La gestión de cuidados a través de este modelo está por determinar una nueva faceta en las unidades de atención médica. Aunque el pronóstico y la supervivencia de los niños prematuros está relacionado directamente con la edad gestacional y peso al nacer, es importante la participación de los profesionales de la salud considerar la implementación de programas de seguimiento como objeto de investigación del recién nacido prematuro dado de alta, con el fin de verificar los efectos benéficos de las intervenciones tempranas en neonatos, los resultados pueden contribuir a evaluar las intervenciones y mejorar el cuidado. (Mata, Salazar, \& Herrera, 2009)

\section{Bibliografia.}

Arandia, R., \& Ayala, M. (2010). RECIEN NACIDO "PREMATURO TARDÍO" FRENTE A LOS RIESGOS QUE DEBEN TENERSE ENCUENTA. Gaceta Médica Boliviana (33), N2, 6469.

Díaz, V. (2005). Lactancia materna: evaluación nutricional en el recién nacido. Revista Cubana de Pediatría, vol 77. N2, http://scielo.sld.cu/scielo.php?script=sci_arttext\&pid=S003475312005000200005 .

Lizarazo-Medina, J. P., Díaz, O., M., J., \& Ariza Riaño, N. E. (2012). Programa madre canguro: una alternativa sencilla y costo eficaz para la protección de los recién nacidos prematuros o con bajo peso al nacer. Revista de Salud Pública, 32-45.

Martínez Contreras, A., Soria Rodríguez, C., Prince Vélez, R., Clark Ordoñez, I., \& Medina Ramírez, M. (2008). 398GinecoloGíay obstetriciade MéxicoPreeclampsia: principal factor de riesgo materno para bajo peso del recién nacido pretérmino. GinecoloGíay obstetriciade México, 398-403.

Mata, M., Salazar, M., \& Herrera, L. (2009). Cuidado enfermero en el recién nacido prematuro. Revista Enfermería Instituto Mexicano de Seguro Social, 45-54. 
Angela R. Briones-Mera; Krissia K. Saltarén-Pérez; Leonardo J. Moreira-Rivas; Jenniffer M. Chavez-Velasquez; Joffre P. Cevallos-Rosales; Mario W. Pacheco-Moreira

Sarmiento Portal, Y., Crespo Campos, A., Portal Miranda, M., Morales Delgado, I., \& Piloña Ruíz, S. (2009). Análisis de la morbilidad y mortalidad en recién nacidos con peso inferior a 1500 g Revista Cubana de Pediatría, http://scielo.sld.cu/scielo.php?script=sci_arttext\&pid=S0034-75312009000400002.

Velázquez Quintana, N. I., Masud Yunes Zárraga, J. L., \& Ávila Reyes, R. (2004). Recién nacidos con bajo peso; causas, problemas y perspectivas a futuro. Boletín médico del Hospital Infantil de México. Vol 1., 73-86. 